\title{
Protonation and monolayer aggregation studied by second-harmonic generation
}

\author{
R. Steinhoff, L. F. Chi, G. Marowsky, and D. Möbius \\ Max-Planck-Institut für Biophysikalische Chemie, Am Fassberg, D-3400 Göttingen, Federal Republic of \\ Germany
}

Received October 14, 1988; accepted December 15, 1988

\begin{abstract}
Various types of aggregates have been studied in Langmuir-Blodgett-type monolayers of selected pyridine and hemicyanine dyes. Environmental changes such as protonation or admixture of chromophores with opposite donor-acceptor characteristics result in dramatic changes of the measured surface susceptibility $\bar{\chi}_{s}^{(2)}$. Both enhanced $\bar{\chi}_{s}^{(2)}$ values in $\mathrm{H}$ aggregates following the shape of the linear absorption band and vanishing $\bar{\chi}_{s}^{(2)}$ values of antiparallel dimers or aggregates have been observed. The experimental results suggest that linear and nonlinear measurements need to be considered for a correct interpretation of the experimental results on monolayer aggregation.
\end{abstract}

\section{INTRODUCTION}

In recent years a growing interest in materials with large second-order nonlinear susceptibility has developed. Organic materials seem to be especially promising candidates for future optoelectronic applications. For an actual data compilation and recent review see Ref. 1. For the qualitative characterization of such materials with respect to nonlinear susceptibility $\bar{\chi}_{s}^{(2)}$ and nonlinear hyperpolarizability $\bar{\alpha}^{(2)}$, the method of second-harmonic generation (SHG) in monolayers deposited onto SHG-inactive surfaces, such as fused quartz, is now well established. However, it should be pointed out that the determination of microscopic molecular quantities such as the essential tensor component of $\bar{\alpha}^{(2)}$, $\alpha_{z^{\prime} z^{\prime} z^{\prime}}^{(2)}=\beta$, is by no means straightforward. Actually, this relation is already a simplification because the $\beta$ value derived from electric-field-induced $\mathrm{SHG}^{1}$ contains, besides the component $\alpha_{z^{\prime} z^{\prime} z^{\prime}}^{(2)}$, several spatially averaged contributions. In addition, all surface SHG measurements ${ }^{2-5}$ start from the assumption that $\alpha_{z^{\prime} z^{\prime} z^{\prime}}^{(2)}$ is the only nonvanishing component of the molecular hyperpolarizability tensor. Also, localfield corrections and molecular interactions are neglected; these two assumptions are generally not true for aggregates. 6,7 In particular, H-type aggregates, because of their narrow-band spectral concentration at the short-wavelength side of the linear monomer absorption band, ${ }^{7,8}$ are attractive sources for large nonlinearities. Because both aggregates and monomers occur in the same monolayer with $a$ priori unknown concentration ratios and unknown absorption cross section of molecules in the aggregate phase, the derivation of molecular nonlinear properties from such a twocomponent system is complicated. Consequently we would like to demonstrate that a combined study of linear absorption measurements and SHG of monolayers leads to a better understanding of such systems.

\section{EXPERIMENT}

All monolayer samples were first prepared on aqueous subphases by standard Langmuir-Blodgett (LB) techniques and were subsequently transferred onto solid substrates from fused quartz. Linear absorption measurements for various angles of incidence and under $s$ - and $p$-polarized excitation were performed with a monolayer absorption spectrometer. ${ }^{9}$ Measurements under normal incidence served to check for the absence of rotational anisotropies. The dichroic absorption spectra of Figs. 1-3 were taken under an angle of incidence of $45^{\circ}$. This procedure was also used for the calibration of the surface density $N_{s}$ of the samples of the protonation series (see Subsection 3.A); samples were prepared by spreading acidified dye solution in isopropanol on purified quartz plates, using cotton wads. For the sake of simplicity, all susceptibility data refer to a coverage density $N_{s}=2.5 \times 10^{14} \mathrm{~cm}^{-2}$.

The nonlinear-optical measurements were carried out with a tunable dye laser pumped by an excimer laser and a $\mathrm{Nd}$ :YAG laser operating at its fundamental wavelength $(1064 \mathrm{~nm})$. The dye laser provided pulses of $15 \mathrm{nsec}$, with a pulse energy of 3-20 mJ, depending on the wavelength of operation (700-940 nm). The Nd:YAG laser pulses lasted 4 nsec with 12-mJ energy. As reference samples for the nonlinear measurements we used the two hemicyanine derivatives, compounds 1 and 2 , with the following chemical structure formulas and $\beta$ and $\chi_{y z y}^{(2)}$ values measured at $1064 \mathrm{~nm}$ (Ref. 10):
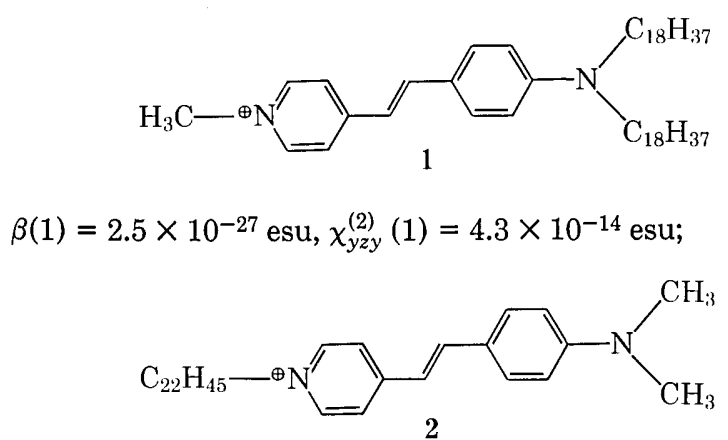

$\beta(2)=2.3 \times 10^{-28} \mathrm{esu}, \chi_{y z y}^{(2)}(2)=: 9.8 \times 10^{-14} \mathrm{esu}$. Both susceptibilities were derived from the expression 


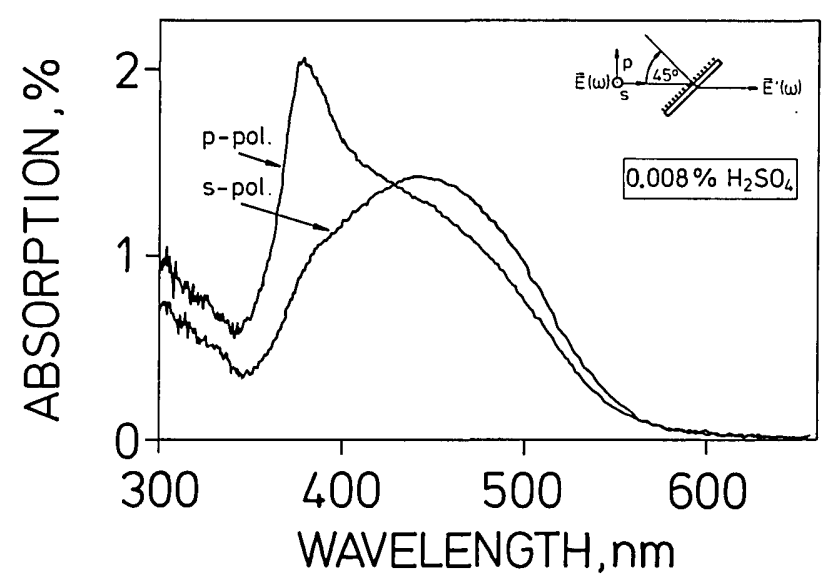

Fig. 1. Dichroic linear absorption spectrum of HAPP-covered LB samples with vanishing nonlinearity after $0.008 \% \mathrm{H}_{2} \mathrm{SO}_{4}$ addition. Inset shows the definition of $s$ - and $p$-polarized excitation geometry.

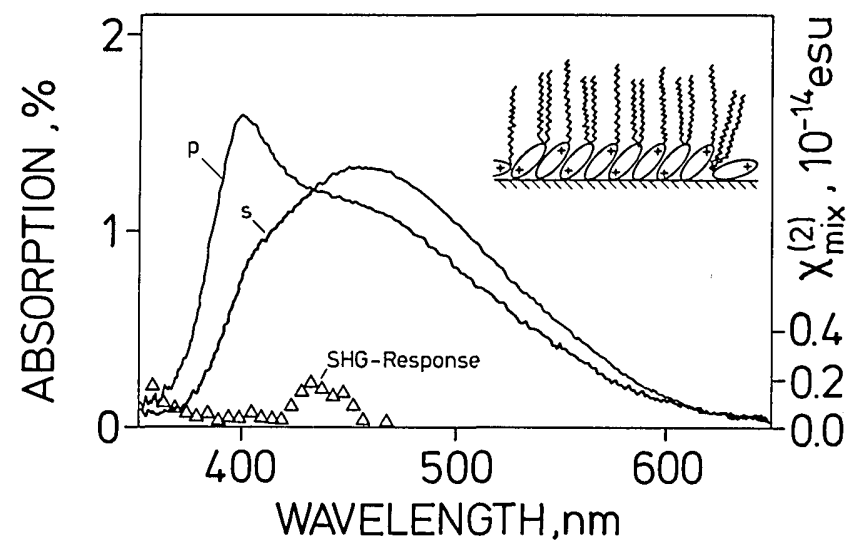

Fig. 2. Dichroic absorption spectra of hemicyanine monolayer mixture together with the SHG response. The inset shows the schematically assumed arrangement of both dyes.

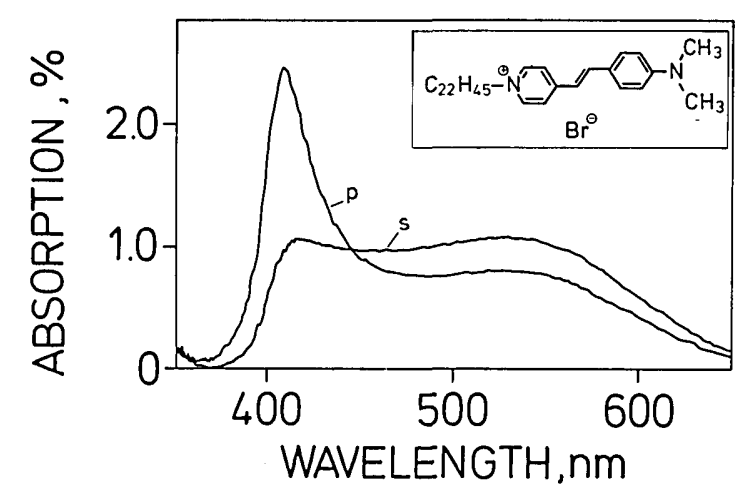

Fig. 3. Dichroic absorption spectra of hemicyanine monolayers forming $\mathrm{H}$ aggregates with peak absorption at $405 \mathrm{~nm}$. Inset shows chemical structure formula.

$$
\chi_{y z y}^{(2)}=\chi_{x z x}^{(2)}=\chi_{z x x}^{(2)}=\chi_{z y y}^{(2)}=1 / 2 N_{s} \sin ^{2} \vartheta \cos \vartheta \beta,
$$

with $\vartheta$ denoting an average tilt angle between the molecular $z^{\prime}$ axis and the surface normal.4,5,11,12

Hemicyanine monolayers showed, under linear and nonlinear tilt angle analysis, rather large values for $\vartheta$ for monomer orientation and a strong tendency for the upright posi- tion for molecules in $\mathrm{H}$ aggregates. The occurrence of measurable aggregation critically depended on the preparation. All LB layers were transferred from the surface of pure Milli-Q-water with a transfer speed of typically $1 \mathrm{~cm} / \mathrm{min}$ and a surface pressure of $30-40 \mathrm{mN} / \mathrm{m}$. The formation of $\mathrm{H}$ aggregates could be enhanced by compression of the dye film immediately after spreading the dye solution. Also, the composition of the subphase was found to play a critical role for $\mathrm{H}$-aggregate formation. Obvious differences in nonlinear properties ${ }^{7,12-14}$ of hemicyanines may be explained with different conditions of monolayer preparation.

\section{RESULTS}

We now present three selected experimental examples, in which aggregation plays a major role for monolayer characterization.

\section{A. Protonation Studies}

In Ref. 7 it was demonstrated that the nonlinearity of hemicyanine monolayers prepared from compound 1 could not be increased by protonation. A systematic investigation of other substances previously characterized with respect to their nonlinear behavior ${ }^{11}$ revealed that the pyridine derivative hexylaminophenylethenylpyridine (HAPP), with the chemical structure

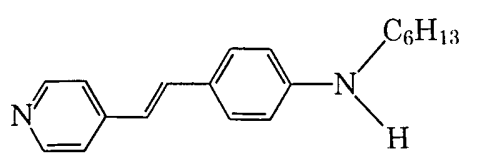

shows, in linear absorption, both an increase and a decrease of the absorption band near $500 \mathrm{~nm}$ when protonated by $\mathrm{HCl}$ addition in liquid solution (Fig. 4). Hence we expected a concomitant increase and decrease of its nonlinearity because of changes in the resonant enhancement of the secondharmonic radiation on excitation with the dye laser at 870 $\mathrm{nm}$. In fact, the second-order susceptibility $\bar{\chi}_{s}^{(2)}$ should follow the shape of the linear absorption $\alpha^{(1)}$ at frequency $2 \omega .^{2}$

Figure 5 shows the dependence of $\sqrt{I_{45^{\circ}, s}{ }^{\mathrm{SH}}}$ versus the protonation-induced absorption at $435 \mathrm{~nm}$ of the (solid) dye film. Addition of $\mathrm{H}_{2} \mathrm{SO}_{4}$ rather than $\mathrm{HCl}$ resulted in morereproducible monolayer absorption data because the presence of water in $\mathrm{HCl}$ caused inhomogeneous dye layers.

$I_{45^{\circ}, s} \mathrm{SH}$ denotes the measured SHG signal under excitation with a fundamental wave polarized at $45^{\circ}$ with respect to the plane of incidence, an angle of incidence of $45^{\circ}$, and $s$-polarized SHG detection. Because $I_{45^{\circ}, s} \mathrm{SH}$ is proportional to $\left|\chi_{y z y}^{(2)}\right|^{2}$ and this susceptibility component follows $\alpha^{(1)}(2 \omega)$, we expect a linear dependence of the square root of the secondharmonic intensity versus protonation-induced absorption. This is in fact the case, as shown in Fig. 5. Also given in this figure are experimental data for $\chi_{y z y}^{(2)}$, taking into account a constant tilt angle of $66 \pm 5^{\circ}$ and an approximate coverage density $N_{s}=2.5 \times 10^{14} \mathrm{~cm}^{-2}$. Addition of $0.003 \% \mathrm{H}_{2} \mathrm{SO}_{4}$ to the solution of HAPP before the solvent is evaporated on the quartz substrate apparently raised the susceptibility $\bar{\chi}_{s}^{(2)}$ by a factor of 4.7. Note that the addition of $0.003 \% \mathrm{H}_{2} \mathrm{SO}_{4}$ to a $5 \times 10^{-4} \mathrm{M}$ solution of HAPP in propanol corresponds to a HAPP: $\mathrm{H}_{2} \mathrm{SO}_{4}$ ratio of $1: 1$. The value for $\bar{\chi}_{s}^{(2)}$ remained constant to as great as $0.006 \%$ addition of $\mathrm{H}_{2} \mathrm{SO}_{4}$. Further 


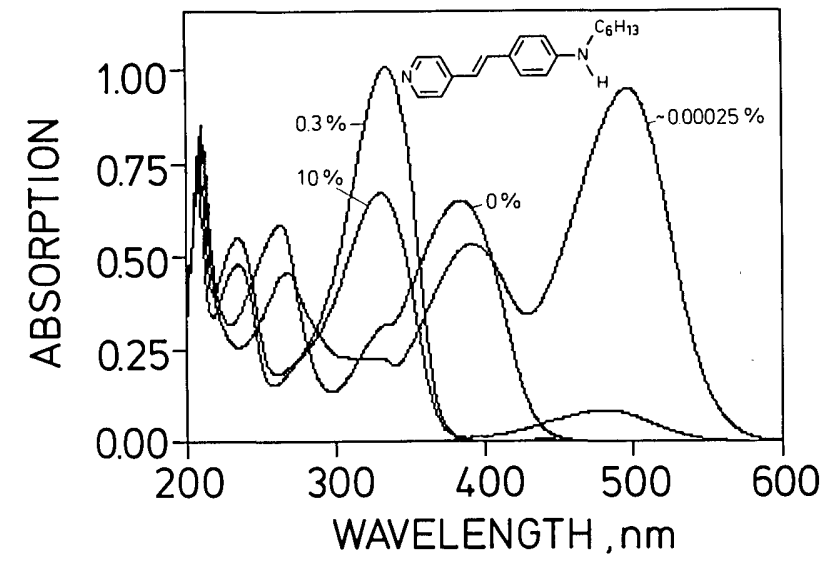

Fig. 4. Linear absorption spectra of HAPP in a propanol solution with the addition of various percentages of $\mathrm{HCl}$.

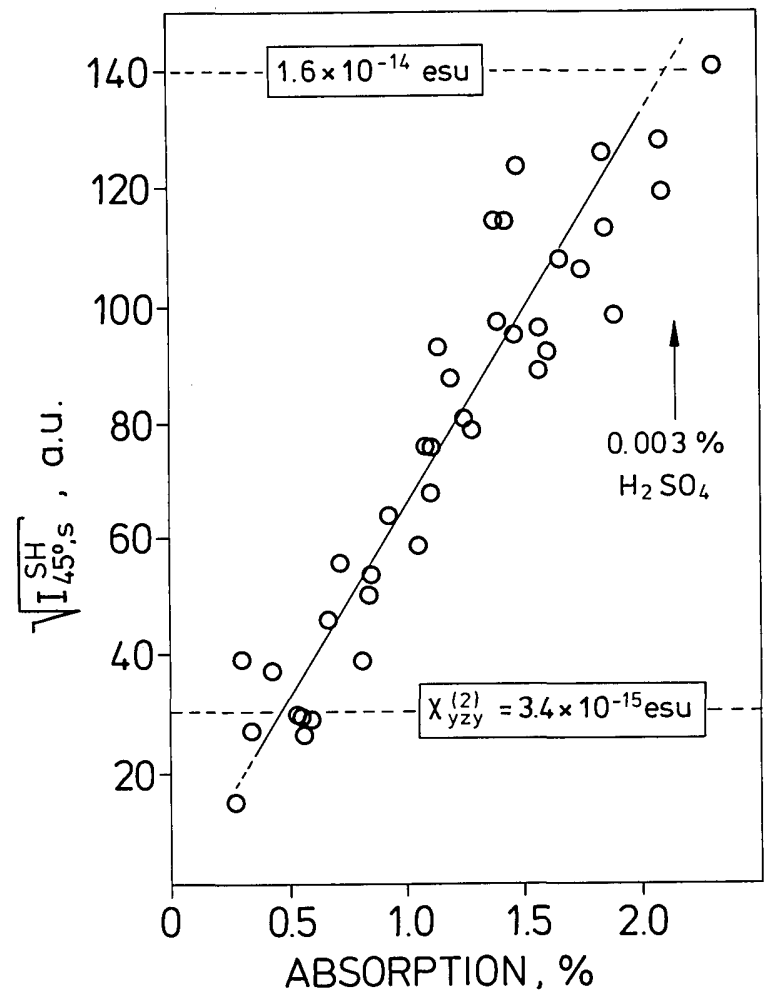

Fig. 5. Square root of selected SHG signals versus protonationinduced absorption of a LB film at $500 \mathrm{~nm}$. Susceptibility data for unprotonated dye monolayer and for protonation by $0.003 \% \mathrm{H}_{2} \mathrm{SO}_{4}$ addition are also given.

protonation led to a surprising effect. In approximately $30 \%$ of the experiments with $\mathrm{H}_{2} \mathrm{SO}_{4}$ addition the nonlinearity $\bar{\chi}_{s}^{(2)}$ dropped by nearly 2 orders of magnitude to $2.3 \times$ $10^{-16}$ esu. In the other cases it remained approximately constant. A comparison of the linear absorption spectra revealed that the drop of the second-harmonic signal was in coincidence with the occurrence of a strong H-type aggregate band at approximately $430 \mathrm{~nm}$. As an example, Fig. 1 shows the dichroic linear absorption spectrum of a sample with almost vanishing nonlinearity after $0.008 \% \mathrm{H}_{2} \mathrm{SO}_{4}$ addition. We conclude that the HAPP molecules in the proton- ated form tend to spontaneous aggregation, leading to inversion-symmetric oligomers.

\section{B. Mixed Monolayers}

The second-harmonic-efficient hemicyanine chromophore 1 has the disadvantage for practical applications of forming monolayers with rather large tilt angles $\left(\vartheta=82^{\circ}\right.$ according to Ref. 10), whereas hemicyanine 2 showed $\vartheta$ values between $42^{\circ}$ (Ref. 12) and $55^{\circ},{ }^{7}$ depending on the actual procedure of LB monolayer preparation. To improve the orientation and to study the influence on the aggregation behavior we tried LB mixtures of both dyes, resulting in dichroic absorption spectra, as shown in Fig. 2, taken for a mixture of 1.4:1.0 of compound 1 to compound 2 and again normalized to a total coverage density $N_{s}=2.5 \times 10^{14} \mathrm{~cm}^{-2}$. The nature of the absorption band centered at $405 \mathrm{~nm}$, which is presumably due to H-type aggregates formed from both constituents of the mixture, has been studied by SHG on excitation at 810 $\mathrm{nm}$. In comparison with the susceptibility of compound 1 at this wavelength and concentration $\left(5.3 \times 10^{-14} \mathrm{esu}\right)$, the nonlinearity of the mixture dropped by a factor of 50 to $\chi_{\mathrm{mix}}^{(2)}$ $=0.1 \times 10^{-14} \mathrm{esu}$, indicating an antiparallel orientation of both chromophores in the aggregate phase, as depicted in the inset of Fig. 2. A systematic variation of the excitation wavelength between 720 and $940 \mathrm{~nm}$ revealed that the resulting susceptibility at the spectral position of the antiparallel aggregates is even lower than the $\bar{\chi}_{s}^{(2)}$ contribution of the remaining monomers that show up near $440 \mathrm{~nm}$ (triangles in Fig. 2).

\section{H Aggregates}

Experimental results concerning the formation of $\mathrm{H}$-type aggregates are presented in Figs. 3 and 6. Figure 3 shows dichroic absorption spectra of monolayers prepared from pure hemicyanine 2. Figure 6 depicts details of nonlinear measurements, indicating that the second-harmonic intensity $I_{p, p}$ SH ( $p$-polarized excitation between 720 and $940 \mathrm{~nm}, p$ -

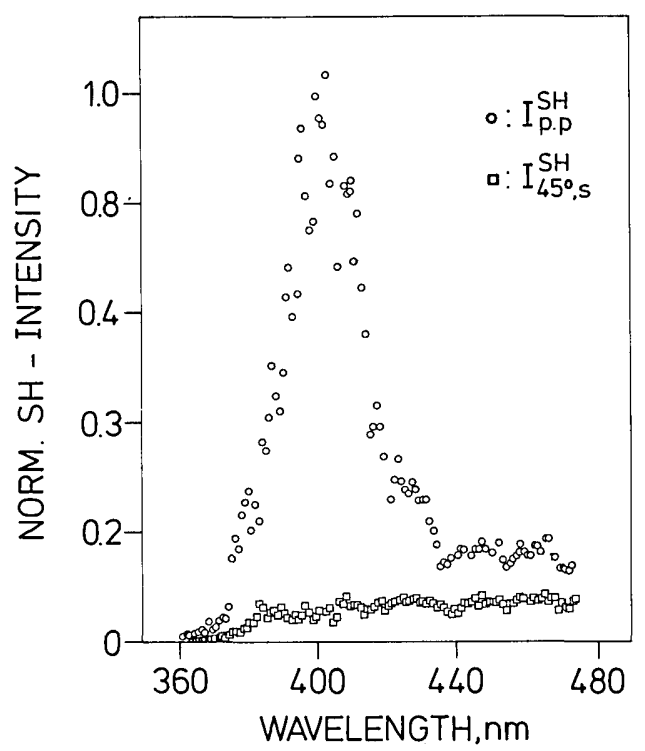

Fig. 6. Nonlinear response of $\mathrm{H}$ aggregates between 360 and 470 $\mathrm{nm}$ for two different second-harmonic intensities, $I_{p, p} \mathrm{SH}$ and $I_{45^{\circ}, \mathrm{s}} \mathrm{SH}$. For details, see the text. 
polarized second-harmonic detection) follows approximately the square of the $p$-polarized linear absorption band centered at $405 \mathrm{~nm}$, as shown in Fig. 3.

The second-harmonic intensity $I_{45^{\circ}, s} \mathrm{SH}$, however, does not follow the shape of the linear absorption at all. Hence the computation of a wavelength-independent tilt angle by the ratio of respective second-harmonic intensities according to Refs. 3, 15, and 16 is impossible. Apparently the two-component system must be characterized by wavelength-dependent fractions $\partial N_{s}(\lambda) / \partial \lambda$ (for monomers) and $\partial N_{s}^{*}(\lambda) / \partial \lambda$ (for aggregates), with

$$
\begin{gathered}
\int_{\Delta \lambda}\left[N_{s}(\lambda) / \partial \lambda\right] \mathrm{d} \lambda=N_{s}, \\
\int_{\Delta \lambda}\left[N_{s}{ }^{*}(\lambda) / \partial \lambda\right] \mathrm{d} \lambda=N_{s}{ }^{*} .
\end{gathered}
$$

The quantities $N_{s}$ and $N_{s}^{*}$ denote the total coverage densities for molecules in monomers and aggregates, respectively, and $\Delta \lambda$ describes the width of the linear absorption band. A wavelength-dependent tilt angle $\vartheta(\lambda)$ could be described by the expression

$$
\vartheta(\lambda)=\frac{N_{s}(\lambda) \vartheta_{0}+N_{s}^{*}(\lambda) \vartheta^{*}}{N_{s}+N_{s}^{*}},
$$

where $\vartheta_{0}$ and $\vartheta^{*}$ denote average tilt angles of narrow distribution functions for both subspecies. Because the wavelength-dependent fractions $N_{s}(\lambda)$ and $N_{s}^{*}(\lambda)$ are difficult to identify from both linear and nonlinear experimental data, we propose a different approach. This approach makes use of the dominant contribution of those components in the susceptibility tensor that are related to the near vertical alignment of molecules in $\mathrm{H}$ aggregates. For this purpose the relation between microscopic polarizability and surface susceptibility can be split into contributions that are due to aggregates $\left(\bar{\chi}_{s}{ }^{*}\right)$ and monomers $\left[\bar{\chi}_{s}^{(2)}\right]$ :

$$
\begin{aligned}
\bar{\chi}_{\operatorname{mix}}^{(2)} & =\bar{\chi}_{s}^{*}+\bar{\chi}_{s}^{(2)} \\
& =N_{s}^{*}\left\langle T_{x y z}^{x^{\prime} y^{\prime} z^{\prime}}\right\rangle^{*} \bar{\alpha}^{*}+N_{s}\left\langle T_{x y z}^{x^{\prime} y^{\prime} z^{\prime}}\right\rangle \bar{\alpha}^{(2)} .
\end{aligned}
$$

The quantity $\left\langle T_{x y z}^{x^{\prime} y^{\prime} z^{\prime}}\right\rangle$ describes the spatially averaged transformation from the molecular coordinate system $x^{\prime}, y^{\prime}, z^{\prime}$ to the laboratory coordinates $x, y, z$. For molecules of rodlike structure and a dominant component $\alpha_{z^{\prime} z^{\prime} z^{\prime}}^{(2)}$, the nonvanishing components of $\bar{\chi}_{s}^{(2)}$ are the components of Eq. (1) together with the following expression for $\chi_{z z z}^{(2)}$ :

$$
\chi_{z z z}^{(2)}=N_{s} \alpha_{z^{\prime} z^{\prime} z^{\prime}}^{(2)} \cos ^{3} \vartheta^{*} .
$$

Experimentally it has been established that near $405 \mathrm{~nm}$ the contributions from $\chi_{s}{ }^{*}$ prevail and should primarily be due to $\chi_{z z z}{ }^{*}=N_{s}^{*}=N_{s}^{*} \alpha_{z^{\prime} z^{\prime} z^{\prime}}^{*} \cos ^{3} \vartheta^{*}$. The angle $\vartheta^{*}$ is the tilt angle of molecules in the aggregate $\alpha_{z^{\prime} z^{\prime} z^{\prime}}^{*}=\beta^{*}(\lambda)$, which is their respective, wavelength-dependent dominant polarizability component with $\beta^{*}(\lambda) \sim \alpha^{(1)}(2 \omega)$.

Linear-optical measurements and the results from Ref. 7 suggest that $N_{s}{ }^{*}=0.5\left(2.5 \times 10^{14} \mathrm{~cm}^{-2}\right)=1.25 \times 10^{14} \mathrm{~cm}^{-2}$. The $\beta$ value for hemicyanine monomers has been determined to be $7.7 \times 10^{-29}$ esu, and the susceptibility $\chi_{z z z}{ }^{*}$ has been determined to be $5.3 \times 10^{-14}$ esu; both data were taken for $405 \mathrm{~nm}$. Hence we can evaluate the ratio $\beta^{*} / \beta$ for various
Table 1. Ratio of $\beta$ Values for Aggregates and Monomers

\begin{tabular}{cc}
$\vartheta^{*}$ & \\
$(\mathrm{deg})$ & $\beta^{* / \beta}$ \\
\hline 0 & 5.5 \\
10 & 5.8 \\
20 & 6.6 \\
30 & 8.4 \\
40 & 12.3 \\
\hline
\end{tabular}

assumptions of the tilt angle $\vartheta^{*}$ (see Table 1$)$. Note that the tensor component $N_{s}^{*} \beta^{*} \cos ^{3} \vartheta^{*}$ is predominant for assumed tilt angles $\vartheta^{*}$ as great as $40^{\circ}$, where, with the inclusion of linear Fresnel factors (Refs. 16 and 17), this contribution is still larger than all other tensor components by a factor of 5. Table 1 also shows that the ratio $\beta^{*} / \beta$ always exceeds 5.5 and that a realistic consideration of $\vartheta^{*}$ fluctuations may lead to a ratio as high as 10 . In fact, enhancement of the molecular hyperpolarizability ratio $\beta^{*} / \beta=6$ was obtained in Ref. 7 from intensity considerations.

\section{SUMMARY AND CONCLUSION}

Three selected examples of aggregation phenomena occurring in LB-type monolayers were studied in detail. It was demonstrated that the application of both linear- and nonlinear-optical techniques is necessary to reveal subtle details of aggregation, such as opposite arrangement of chargetransfer complexes in inversion-symmetric $\mathrm{H}$ aggregates.

Protonation of a selected pyridine derivative leads to a considerable increase in its molecular second-order nonlinearity. The buildup and decrease of protonation-induced absorption bands could be monitored by the concomitant changes in the nonlinear surface susceptibility $\bar{\chi}_{s}^{(2)}$. The most dramatic event was the protonation-induced change from an optimized susceptibility $\chi_{\mathrm{opt}}^{(2)}=1.6 \times 10^{-14} \mathrm{esu}$ (due to $\mathrm{H}_{2} \mathrm{SO}_{4}$ addition by nearly 2 orders of magnitude) to $\bar{\chi}_{s}^{(2)}=$ $2.3 \times 10^{-16} \mathrm{esu}$ (due to spontaneous aggregation into inversion-symmetric oligomers).

The formation of $\mathrm{H}$ aggregates in pure hemicyanine monolayers was studied. The assumptions usually used in surface $\mathrm{SHG}$, such as nonexisting molecule-molecule interactions or the concept of a wavelength-independent dominant tensor component $\alpha_{z^{\prime} z^{\prime} z^{\prime}}^{(2)}$ of the molecular hyperpolarizability tensor, no longer hold in such a two-component system of molecules in monomers and aggregates. In agreement with a previous investigation, we established that the hyperpolarizability per molecule in an aggregate, $\beta^{*}$, exceeds (despite the reduced linear absorption) the $\beta$ value of the monomer at the same wavelength by a factor of at least 6 . Alternatively, we might interpret this result as an indication that under the given experimental conditions six molecules of monomer hyperpolarizability $\beta$ form an aggregate and radiate coherently.

\section{ACKNOWLEDGMENT}

All pyridine and hemicyanine substances were synthesized with high purity by D. Dorsch, Merck/Darmstadt. 


\section{REFERENCES}

1. D. S. Chemla and J. Zyss, eds., Nonlinear Optical Properties of Organic Molecules and Crystals (Academic, New York, 1987).

2. T. F. Heinz, C. H. Chen, D. Ricard, and Y. R. Shen, Phys. Rev. Lett. 48, 478 (1982).

3. T. F. Heinz, H. W. K. Tom, and Y. R. Shen, Phys. Rev. A 28, 1883 (1983)

4. Th. Rasing, G. Berkovic, Y. R. Shen, S. G. Grubb, and M. W. Kim, Chem. Phys. Lett. 130, 1 (1986).

5. G. Marowsky, R. Steinhoff, G. A. Reider, D. Erdmann, and D. Dorsch, Opt. Commun. 63, 109 (1987).

6. M. Orrit, D. Möbius, U. Lehmann, and H. Meyer, J. Chem. Phys. 9, 4966 (1985).

7. G. Marowsky and R. Steinhoff, Opt. Lett. 13, 707 (1988).

8. G. H. Wagnière and J. B. Hutter, Opt. Soc. Am. B 6, 693 (1989).

9. H. Kuhn, D. Möbius, and H. Bücher, in Physical Methods of
Chemistry, A. Weissberger and B. Rossiter, eds. (Wiley, New York, 1972), Vol. 1

10. G. Marowsky, L. F. Chi, D. Möbius, R. Steinhoff, Y. R. Shen, D. Dorsch, and B. Rieger, Chem. Phys. Lett. 147, 420 (1987).

11. G. Marowsky, A. Gierulski, R. Steinhoff, D. Dorsch, R. Eidenschink, and B. Rieger, J. Opt. Soc. Am. B 4, 956 (1987).

12. I. R. Girling, N. A. Cade, P. V. Kolinsky, R. J. Jones, I. R. Peterson, M. M. Ahmad, D. B. Neal, M. C. Petty, G. G. Roberts, and W. J. Feast, J. Opt. Soc. Am. B 4, 950 (1987).

13. J. S. Schildkraut, T. L. Penner, C. S. Willand, and A. Ulman, Opt. Lett. 13, 134 (1988).

14. D. Lupo, W. Prass, U. Scheunemann, A. Laschewsky, H. Ringsdorf, and I. Ledoux, J. Opt. Soc. Am. B 5, 300 (1988).

15. Y. R. Shen, J. Vac. Sci. Technol. B 3, 1464 (1985).

16. B. Dick, A. Gierulski, and G. Marowsky, Appl. Phys. B 38, 107 (1985).

17. V. Mizrahi and J. E. Sipe, J. Opt. Soc. Am. B 5, 660 (1988). 